\title{
Intelligent Spectrum Allocating Scheme in Multi-RATs Networks
}

\author{
Yizhe $\mathrm{Li}^{1}$, Zhonghao Zhang ${ }^{1}$, Lijun Chen ${ }^{2}$, Fuchang $\mathrm{Li}^{1}$ and Yi Feng ${ }^{1}$ \\ ${ }^{1}$ Wireless Technology Research Dep. Network Technology \\ Research Institute, China Unicom, Beijing, China \\ ${ }^{2}$ Research Institute, ZTE corporation, Shanghai, China \\ liyz49@chinaunicom.cn
}

\begin{abstract}
The explosive growth in data traffic is resulting in a spectrum crunch forcing many wireless network operators to look forward refarming their $2 G$ Global System (GSM) and $3 G$ Universal Mobile Telecommunications System (UMTS) spectrum and deploy more spectrally efficient Long Term Evolution (LTE). However, mobile network operators face a challenge when it comes to spectrum refarming because $2 G$ or $3 G$ technologies are still widely used for voice services. And $2 G$ and $3 G$ equipment have long life cycles, e.g. smart meters, and it is expensive to migrate these devices to newer technology since a truck roll will typically be required to the site where a device is deployed. Nevertheless, operators are keen to either force their $2 G$ or $3 G$ customers to migrate so that they can refarm the spectrum or set aside a portion of the $2 G$ or $3 G$ spectrum for continuing operating $2 G$ or $3 G$ and only refarm the rest for LTE. Due to this requirement, future wireless networks should be able to dynamically allocate resources to maintain the quality of service and promote the efficient use of radio spectrum. In this paper, we propose a new intelligent method for allocating spectrum between radio access technologies (RATs) such as GSM, UMTS and LTE. We designed a dynamic spectrum allocation scheme to increase the capacity of high traffic volume RAT without hurt other RATs' performance as well as maximize the spectrum efficiency of the whole network.
\end{abstract}

Keywords: Spectrum allocation, multi-RATs, prediction model, Neural Network

\section{Introduction}

Today, available frequency spectra are assigned to different radio services by following a fixed spectrum allocation policy. With respect to Radio Access Technologies (RATs), this means that there is a dedicated band used exclusively by one RAT operated by a single operator. As broadband radio services are expected to continue their recently observed explosive growth also in the future, it can be foreseen that the demand in additional frequency bandwidth cannot be satisfied any longer by following the conventional spectrum allocation scheme. Hence, new measures to unlock frequency spectrum need to be found.

A suitable measure for that is known under the term of spectrum sharing, where different radio systems are granted a coordinated access to the same spectrum band [1]. Based on the assumption that different radio systems will not have the same demands for bandwidth at the same time, a heavily loaded RAT may borrow frequency resources from another RAT that are temporarily unused. This approach not only relaxes the problem of spectrum shortage, but also significantly improves the efficiency of overall spectrum utilization.

Spectrum sharing between heterogeneous radio services is considered in the context of the utilization of the so-called White Spaces in the frequency spectrum of TV broadcast services [2]. Today, these White Space frequencies are used by secondary access of devices for project making and special events (PMSE); however, their operation is also of 
temporary nature and hence there is a high potential for the use of this spectrum by other radio devices. Opening this spectrum for an opportunistic access of new additional radio devices requires those devices to get aware of the current spectrum usage, allowing them to avoid interferences and potential distortions of the other systems operating in the same band. This can be enabled by the cognitive radio technology. Currently, a lot of research is carried out in this context, and there exist many international collaboration research projects seeking for practical solutions, like the FP7 projects FARAMIR [3], CogEU [4], QoSMOS [5] and ideas, but extends the scope to multiple frequency bands that are available for opportunistic spectrum access.

If multiple different radio services are demanding a limited spectrum resource, decision methods for a suitable resource allocation become of interest. For this use case, spectrum auctioning methods have been developed that try to satisfy a maximum of service requests while maximizing the spectrum utilization [8]-[10]. These techniques are also in the focus of the FP7 project Saphyre [6], which elaborates on various solutions for efficient sharing of physical resources, in particular spectrum. A special scenario for spectrum sharing is an inter-operator scenario where multiple operators are using the same RAT, which allows for a flexible inter-operator resource management. Here, spectrum borrowed to another operator does not necessarily need to be freed, as long as the resources used for the simultaneous access of different operators are orthogonal. Major research in this field has been conducted in the scope of the FP\& E3 project [11] and its predecessors FP6 E2R phase 1 and 2 .

In this paper, we study the resource dynamic sharing problem for the multi-RATs networks. The main contributions in this paper can be summarized as follows:

Firstly, we propose a spectrum sharing system in which multi-RATs can occupy the spectrum band by chance, according to the schedule decision of the central controller. We also study the schedule scheme in detail, including the spectrum band allocation principle, the methods of gain computation and interference estimation.

Then, we introduce an approach of spectrum demand forecast based on neural network, by which the central controller can predict the capacity and interference of each RAT in coming time, and make the resource allocation decision in advance. This approach could help improve the promptness and accuracy of the spectrum allocation, as well as reduce the requirement of real time computation capacity of the controller to increase the feasibility of the system, comparing with [13].

Finally, we conduct a practical test system based on the existing GSM/UMTS/LTE networks. By adding the central controller to the network management system of the existing networks, we verify the effect of the whole spectrum sharing scheme. It may be more credible and comprehensive than simulation of computers.

The remainder of the paper is organized as follows. In Section 2, the proposed spectrum sharing system is presented. In Section 3, we formulate the spectrum allocation problem for multi-RATs and propose the highly efficient algorithm to solve it. The experiment results are presented in Section 4 and the conclusion is drawn in Section 5.

\section{System Model}

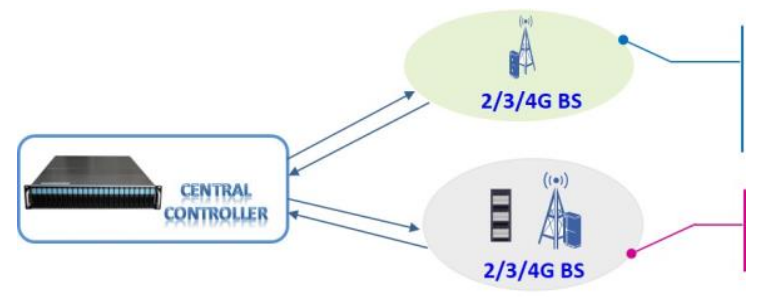

Figure 1. System Model 
As shown in Figure 1, we consider in this paper a spectrum sharing system where two legacy 2/3/4G radio access technologies (RATs), recorded as A and B, are overlaid, the 2G BS means GSM BS, 3G BS means UMTS NodeB and 4G BS means LTE eNodeB. In addition, the whole available spectrum band is $\mathrm{W} \mathrm{MHz},{ }^{W_{A}} \mathrm{MHz}$ is fixed assigned to system $\mathrm{A}$ and $W_{B} \mathrm{MHz}$ is fixed assigned to system $\mathrm{B}, W_{C}=W-W_{A}-W_{B}$ is dynamic spectrum resource and can be dynamically assigned to A or B. To improve frequency resource utility and reduce traffic congestion, $W_{C}$ should be reasonably assigned so that the whole networks' benefits can be maximized.

To realize the dynamic spectrum sharing, we need to integrate a central spectrum manager (CSM) as a new component in the network management system architecture. On one hand, the CSM connect to both RATs to monitor the information on their current conditions periodically, for example, the RATs report their traffic volume in last cycle, as well as spectrum efficiency, allocated bandwidth, received signal power and so on. On the other hand, it make the spectrum allocation decisions of next cycle between A and B based on the analysis results and strategy proposed in the next chapter.

\section{Solution Specification}

\subsection{Goal Function}

The spectrum allocation should maximize the weighted gain of the whole system in the scheduling cycle, recorded as $\mathrm{G}$, which can be calculated as follow:

$$
G=\alpha_{A}\left\lceil G_{A}+\alpha_{B}\left\lceil G_{B}\right.\right.
$$

Where $\alpha_{A}$ and $\alpha_{B}$ are the weights of unit traffic values of RAT A and B, $G_{A}$ is the traffic volume of RAT A and $G_{B}$ is the traffic volume of RAT B in the scheduling cycle. The length of scheduling cycle depends on the traffic changing speed, the more quickly the traffic changes, the shorter the cycle is. In this paper we set the cycle as one hour. In the formula (1), $G_{A}=\gamma_{A} \llbracket W_{A}, G_{B}=\gamma_{B} \llbracket W_{B}=\gamma_{B} \llbracket\left(W-W_{A}\right) . \gamma_{A}$ and $\gamma_{B}$ are respectively RAT A and B's spectrum efficiency weights. $W_{A}$ and $W_{B}$ are respectively the allocated bandwidth of RAT A and B, W is the total bandwidth. Thus the goal function can be rewritten as follows:

$$
\begin{aligned}
& G=\alpha_{A} \llbracket \gamma_{A} \square W_{A}+\alpha_{B} \square \gamma_{B} \square\left(W-W_{A}\right) \\
& =\left(\alpha_{A} \square \gamma_{A}-\alpha_{B} \llbracket \gamma_{B}\right) \square W_{A}+\alpha_{B} \llbracket \gamma_{B} \square W
\end{aligned}
$$

The $\alpha_{A}$ and $\alpha_{B}$ represent the importance of different RATs' traffic and are assignment by Operators, for example, if RAT A is GSM and B is LTE, the price of 1MB traffic of LTE is half of GSM, so the $\alpha_{B}$ can be set to half of $\alpha_{A}$. Because the traffic values are relatively fixed, $\alpha_{A}$ and $\alpha_{B}$ are not changing in several consecutive scheduling cycles. But due to the rapid change of traffic, $\gamma_{A}$ and $\gamma_{B}$ change for each cycle. $\gamma_{A}$ is decided by $W_{A}$ and $T_{A}, T_{A}$ is the expected traffic volume of RAT A of this cycle, thus $\gamma_{A}$ can be represented as a function of $W_{A}$ and $T_{A}, \gamma_{A}=F_{A}\left(W_{A}, T_{A}\right), \gamma_{B}$ can be represented ${ }_{\text {as }} \gamma_{B}=F_{B}\left(W_{A}, T_{B}\right)$ 


$$
F_{A}=\left\{\begin{array}{l}
\bar{\gamma}_{A}, T_{A}>\bar{\gamma}_{A} * W_{A} \\
\frac{T_{A}}{W_{A}}, \text { else }
\end{array} \quad F_{B}=\left\{\begin{array}{l}
\bar{\gamma}_{B}, T_{B}>\bar{\gamma}_{B} *\left(W-W_{A}\right) \\
\frac{T_{B}}{W-W_{A}}, \text { else }
\end{array}\right.\right.
$$

So if the $T_{A}$ and $T_{B}$ are determined, the goal function will be a reciprocal function and the maximum value can be found, as well as the best spectrum allocation decision could be made, which is how much $W_{A}$ is.

To determine the $T_{A}$ and $T_{B}$ in the scheduling cycle, we need to predict the traffic volume based on the previous data. In this paper we use neural network method to build a prediction model.

\subsection{Prediction Model}

There have been a number of works studied on user and traffic predicting methods [1415]. The previous works have tried to predict the development of the traffic of electronic commerce according to historical traffic volume [14], but the traffic of mobile network is more difficult to predict because there are many factors affecting the traffic, such as user number, network resource utilization, coverage quality and so on. The concrete method used in predicting mobile network traffic in this paper is also different from the previous works, in [15] the authors forecasted the network evolution trend using Markov chain, but the method cannot be used in mobile network due to the traffic development is highly related to the historical data.

Back propagation (BP) neural network $(\mathrm{NN})$ is a kind of multilayer feed forward neural network [16-20], usually has one or more hidden layers and one output layer, its transfer function is a $S$ function that can be carried everywhere, as well as it has a strong mapping ability and can be used to approximate any nonlinear function. In the BP neural network, the signal propagation is moving forward, and error propagates backward from the last output layer in the opposite direction of the input conveyor after contrasting with the expected outputs.

We use the NN to build a prediction model in order to predict the user number and traffic volume of the networks. Based on the big data analysis, we found that the development of GSM/UMTS/LTE networks represented some regularity, including: longterm regularity, which meant that the user number was influent by the environment, society, market, economy and had obvious statistical regularity; short-term regularity, which meant that the traffic volume changed fast and hard to found statistic rules. But we know the traffic volume is influent by user number, traffic model and the network augments, due to the stability of those influence factors, we still can find the regularity of traffic volume based on $\mathrm{NN}$ model by taking the stable influence factors into account. The $\mathrm{NN}$ model has great fault tolerance and robustness, as well as nonlinear approximate ability, thus is very suitable for large-scale information forecasting. So we use the historical data of every cell's statistical network index data in every hour of several days to construct input vector of input layer, including user number $U_{n}$, uplink and downlink PRB utilization $P_{D}$ and $P_{U}$, user data rate $R_{U}$, cell throughput $T_{C}$, average CQI of cell, and so on. The input vector $X=\left(U_{n}, P_{D}, P_{U}, R_{U}, T_{C}\right.$, CQI...). And the hidden layer was built to complete an overall nonlinear analysis of the input data. Considering the abundant data we set the number of hidden layer to 1 . Because the hidden layer used S-function, the output of this layer was $y=f(n e t)=1 / 1+e^{-n e t}$. In the neural network, the key process is learning, which made the mean square value be constantly reduced to the desired value. 
We used steepest decent method and the iterative formula is [21-22] : ${ }_{k+1}=w_{k}-a_{k} g_{k}$, where the $w_{k}$ is the coefficient between input layer and hidden layer, as well as between hidden layer and output layer. $w_{k+1}$ is the coefficient after next iteration, ${ }^{a_{k}}$ represented the learning rate of the network, ${ }_{k}$ represented the current error gradient of the function.

We use the NN based prediction model to predict the network A's traffic volume $T_{A}$ and network B's traffic volume $T_{B}$. According to our analysis, if we input three consecutive days' information of A as input vector, including user number, average RSRP, average SINR, average $T_{A}$, average downlink rate of some hour in every day, we can predict accurately the network's traffic volume $T_{A}$ of the same hour in 4th day, and the accuracy is above $80 \%$. So we can use this model to predict network A and B's traffic $T_{A}$ and $T_{B}$, in order to the compute the value $W_{A}$ in (2).

\section{Experimental Results}

To demonstrate the advantage of our scheme, a large scale experiment was conducted in the real network of city Q. In this area GSM and LTE networks coexist and cocoverage, the customary spectrum allocation approach is shown in Figure 2. According to the statistical data the busy time of GSM networks appear at daytime because the users mainly require voice call services in work time, and at night people more often browse web, watch video, use instant messenger, so the LTE networks are more busy and GSM's spectrum will be relative free. Thus we implement the intelligent spectrum allocation scheme between GSM and LTE networks in city Q.

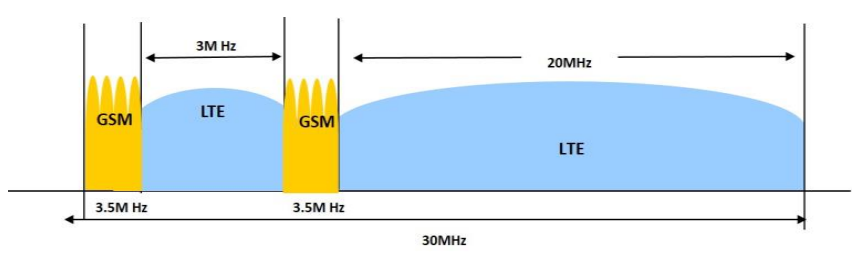

Figure 2. Customary Spectrum Allocation Scheme

The experiment network contains 12 LTE sites and 66 cells, as well as 7 GSM sites and 12 cells, shown in Figure 3.

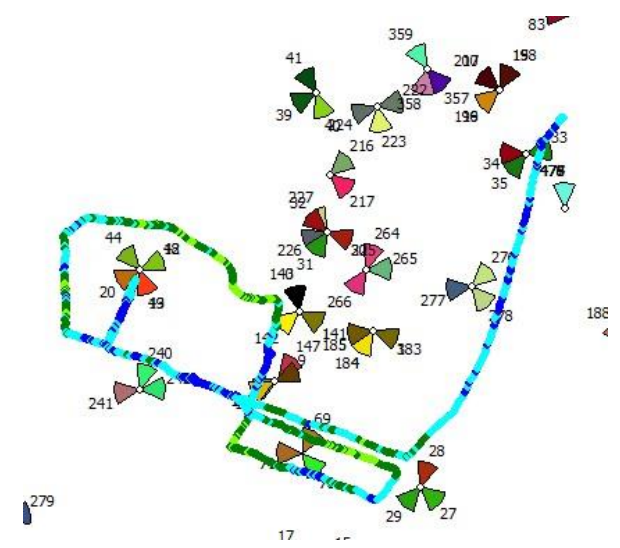

Figure 3. Experiment Network 
The GSM and LTE networks' information data were collected and reported to CSM, including user number, average RSRP, average SINR, average $T_{A}$, average downlink rate of every hour in three consecutive days, and were input into the trained $\mathrm{NN}$ prediction model as input vector, the fourth day's traffic volume of every hour was output from the model. We predicted 7 days' traffic and the accuracy rates were as follows:

Table 1. Traffic Volume Prediction

\begin{tabular}{|l|l|l|l|l|l|l|l|}
\hline Day & 4 & 5 & 6 & 7 & 8 & 9 & 10 \\
\hline Accuracy rate (\%) & 81.2 & 91.1 & 80.0 & 83.3 & 87.1 & 81.6 & 80.0 \\
\hline $\begin{array}{l}\text { Average accuracy } \\
\text { rate (\%) }\end{array}$ & \multicolumn{7}{|c|}{83} \\
\hline
\end{tabular}

The predicted traffic volume of GSM and LTE were input into formula (2) and (3) as $T_{A}$ and $T_{B}$, and Other parameters are as follow: $\gamma_{A}=1(/ \mathrm{Hz} / \mathrm{s}), \quad \gamma_{B}=2.5(/ \mathrm{Hz} / \mathrm{s})$,

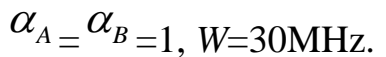

Thus the only unknown parameter was $W_{A}$ and the goal function would be a reciprocal function and the $W_{A}$ value which would be maximize the goal $\mathrm{G}$ can be found. According to the calculation results, $W_{A}=7 \mathrm{MHz}$ as well as $W_{B}=23 \mathrm{MHz}$ from 8.am to 8.pm, which means the bandwidth of GSM is $7 \mathrm{MHz}$ as well as bandwidth of LTE carrier 1 and carrier 2 were respectively $20 \mathrm{MHz}$ and $3 \mathrm{MHz}$, and $W_{A}=5 \mathrm{MHz}$ and $W_{B}=25 \mathrm{MHz}$ from $8 . \mathrm{pm}$ to 8.am, which means the bandwidth of GSM is $5 \mathrm{MHz}$ as well as bandwidth of LTE carrier 1 and carrier 2 were respectively $20 \mathrm{MHz}$ and $5 \mathrm{MHz}$.

The experiment was divided into three stages, in the first stage, the GSM band was located on both sides of LTE carrier 2, as shown in Figure 4 and Figure 5.

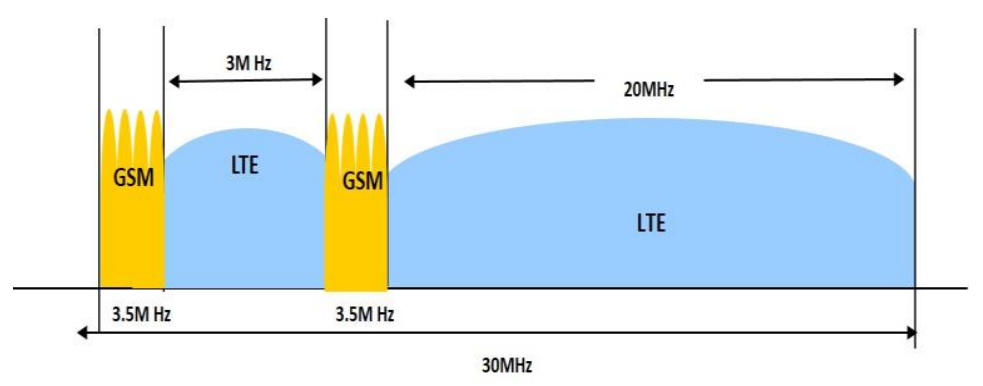

Figure 4. Spectrum Allocation from 8.am to 8.pm

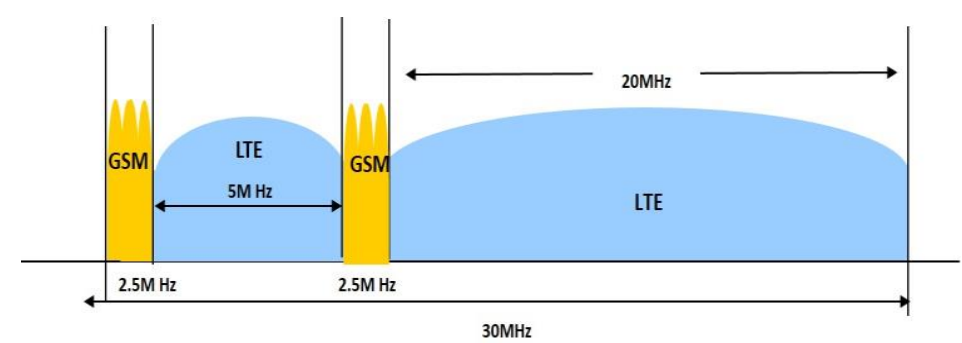

Figure 5. Spectrum Allocation from 8.pm to 8.am 
The result data showed in 4th day the GSM traffic volume changed from 50\% to $25 \%$ after 8.pm as shown in Figure 6, and the dynamic spectrum allocation was triggered, bandwidth of LTE carrier 2 changed from $3 \mathrm{MHz}$ to $5 \mathrm{MHz}$, as shown in Figure 7. And GSM traffic volume significantly increased at 8.am as well as the bandwidth of LTE carrier 2 changed from $5 \mathrm{MHz}$ to $3 \mathrm{MHz}$.

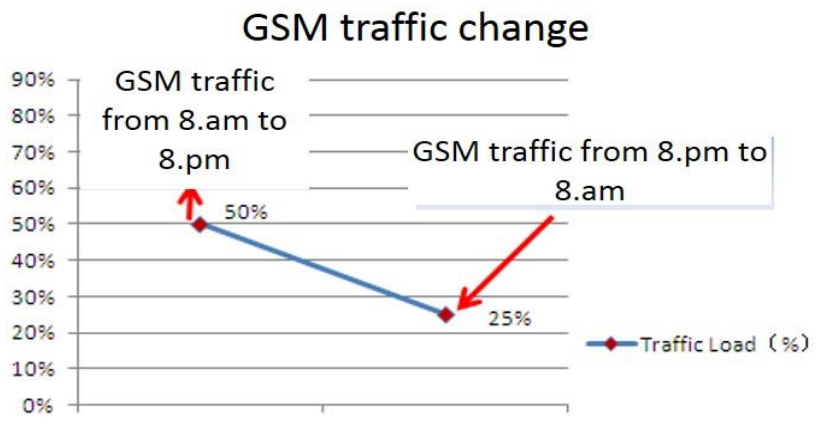

Figure 6. GSM Traffic Change at 8.pm

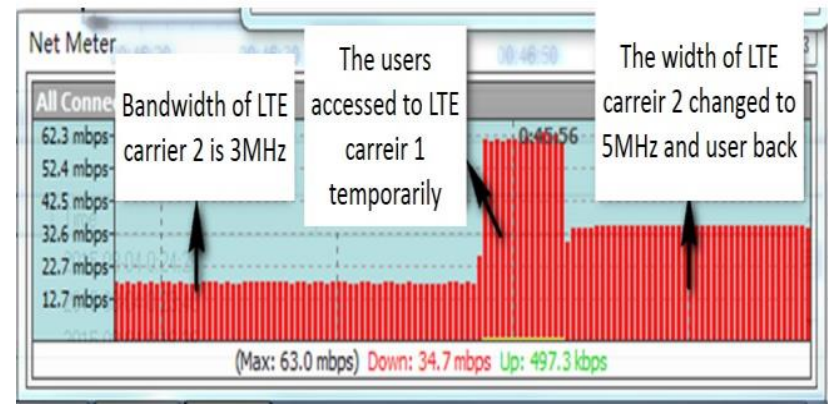

Figure 7. Bandwidth of LTE Carrier 2 Changed

After bandwidth changed from $3 \mathrm{MHz}$ to $5 \mathrm{MHz}$, the downlink capacity of LTE carrier 2 increased from $17.54 \mathrm{Mbps}$ to $35 \mathrm{Mbps}$, and uplink capacity increased from $7.45 \mathrm{Mbps}$ to 13.01Mbps as shown in Table 2.

Table 2. LTE Network Parameters Change

\begin{tabular}{|l|l|l|l|l|l|l|l|}
\hline $\begin{array}{l}\text { LTE } \\
\text { bandwidth }\end{array}$ & downlink & uplink & SINR & RSRP & RRC drop rate & $\begin{array}{l}\text { ERAB } \\
\text { drop rate }\end{array}$ & $\begin{array}{l}\text { Handover } \\
\text { success }\end{array}$ \\
\hline $3 \mathrm{MHz}$ & $17.54 \mathrm{Mbps}$ & $7.45 \mathrm{Mbps}$ & 12.75 & -82.07 & $0.00 \%$ & $0.00 \%$ & $100 \%$ \\
\hline $5 \mathrm{MHz}$ & $35 \mathrm{Mbps}$ & $13.01 \mathrm{Mbps}$ & 13.11 & -82.57 & $0.00 \%$ & $0.00 \%$ & $100 \%$ \\
\hline
\end{tabular}

The network indexes of GSM were shown in Table 3 and the network worked well after spectrum reallocation.

Table 3. GSM Network Parameters Change

\begin{tabular}{|l|l|l|l|l|l|}
\hline & $\begin{array}{l}\text { Resource } \\
\text { usage }\end{array}$ & $\begin{array}{l}\text { Call } \\
\text { success }\end{array}$ & $\begin{array}{l}\text { Call } \\
\text { setup } \\
\text { time(s) }\end{array}$ & $\begin{array}{l}\text { Dr } \\
\text { op } \\
\text { rate }\end{array}$ & $\begin{array}{l}\text { RQ } \\
0 \sim 3 \\
\text { rate }\end{array}$ \\
\hline $8 . \mathrm{am}$ & $50 \%$ & $100 \%$ & 6.168 & $0 \%$ & $100 \%$ \\
\hline $8 . \mathrm{pm}$ & $25 \%$ & $100 \%$ & 6.029 & $0 \%$ & $100 \%$ \\
\hline
\end{tabular}


According to the experiment results, after spectrum dynamic allocation by the proposed method, the uplink throughput of LTE network was increased by $80 \%$ and downlink throughput was increased by $100 \%$, as well as the average uplink and downlink traffic volume of LTE network in one day were increased by $100 \%$ and $200 \%$. The networks' key indexes such as call drop rate, handover success rate and so on maintained a good level, and the spectrum efficiency of the whole bandwidth was increased by $63.9 \%$.

In the second stage of the experiment, we implemented the fine-grained quarter level spectrum allocation based on the hour level spectrum allocation of first stage. From 8.am to 8.pm, the GSM and LTE networks' traffic of each half hour was predicted, and it can be seen there were $2 / 3$ of quarters between 8.am to 8.pm the GSM traffic volume $T_{A}$ significantly decreased and the LTE traffic volume $T_{B}$ increased, such as from 12:45 am to $1: 00 \mathrm{pm}, 1: 45 \mathrm{pm}$ to $2: 00 \mathrm{pm}$ and so on, which could be called LTE busy time. So during these quarters the $W_{A}$ was recalculation according to (2) and (3) by bring the predicted $T_{A}$ and $T_{B}$ of the quarters into the formulas, and the results are $W_{A}=5 \mathrm{MHz}$ and $W_{B}=25 \mathrm{MHz}$, to reduce the frequency of spectrum allocation and the user switching rate caused by it, we allocated the spectrum between GSM and LTE as shown in Figure 8 from 8.am to 8.pm, $2 \mathrm{MHz}$ GSM band was insert into LTE carrier 1 and the LTE RBs of that $2 \mathrm{MHz}$ band were blocked in most of the time. But during the LTE busy time, the inserted 2MHz GSM band was returned to LTE carrier 1 as shown in Figure 9.

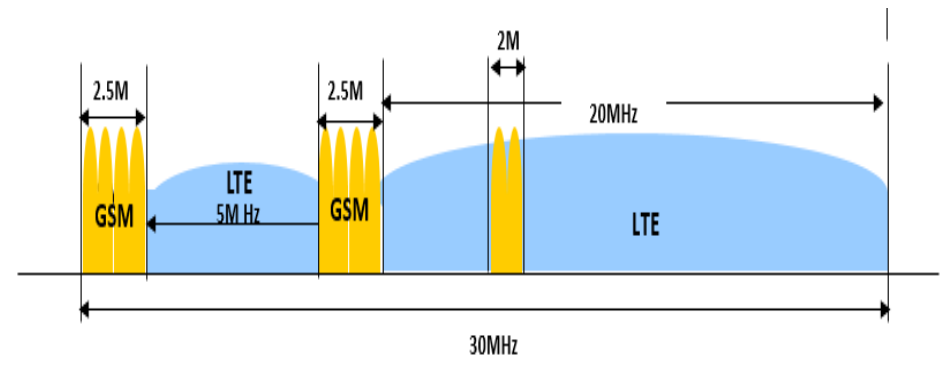

Figure 8. Spectrum Allocation from 8.am to 8.pm in Stage 2

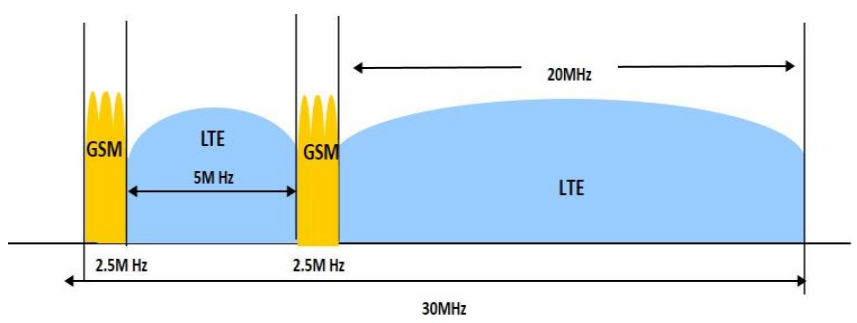

Figure 9. Spectrum Allocation During LTE Busy Time

By using the fine-grained spectrum allocation scheme proposed in stage 2, the LTE network could use more idle spectrum resource of GSM and the average peak throughput of LTE carrier 1 during 8.am to 8.pm can be improved by 5\% as shown in Figure 10. And the spectrum efficiency of the $2 \mathrm{MHz}$ spectrum dynamically shared by GSM and LTE can be improved by $100 \%$ as shown in Figure 11. 


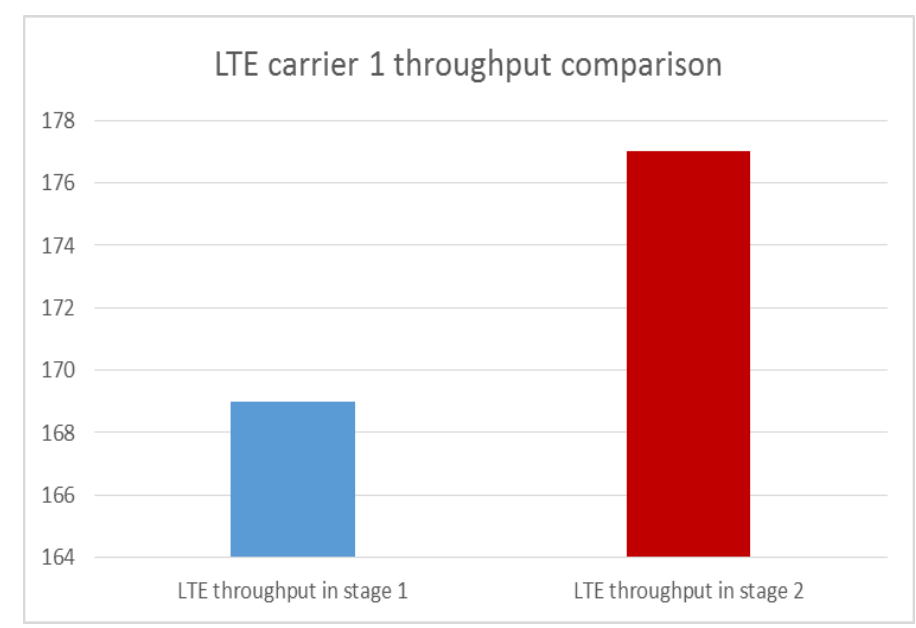

Figure 10. LTE Throughput Comparison between Stage 1 and 2

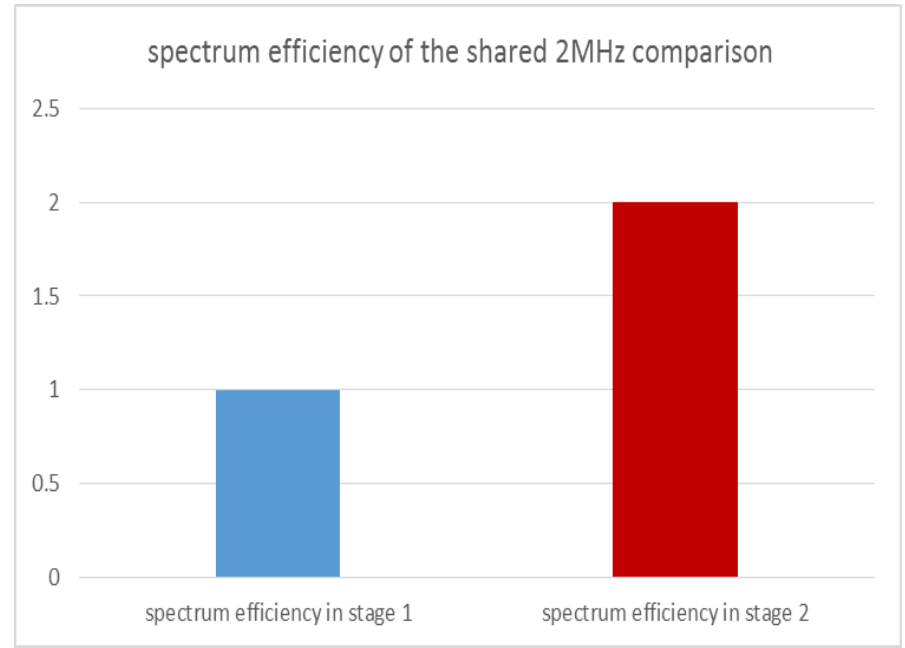

Figure 11. Spectrum Efficiency Comparison between Stage 1 and 2

Therefore, the proposed methodology could increase the capacity of high traffic volume RAT without hurt other RATs' performance by intelligently allocating spectrum to different RATs, as well as maximize the spectrum efficiency of the whole system.

\section{Conclusion}

In this paper, we have presented a new intelligent method for allocating spectrum between RATs in an co-coverage scenario. The presented methodology consists of two complimentary parts, the traffic volume prediction and spectrum optimal allocation. The traffic volume prediction represents how to predict future traffic volume of each RAT based on NN model and historical data, in order to provide parameters for spectrum allocation scheme; and the spectrum optimal allocation makes the decision of allocating spectrum to each RAT according to the calculation results of goal function. To demonstrate the advantages of the method, we carried out experiment in real networks. The results illustrate that with applying the proposed intelligent spectrum management we can effectively increase the capacity of the RATs whose traffic volume increase as well as ensure other RATs' performance. Furthermore, the capacity of the whole network and the spectrum efficiency will be maximized. The experiment results confirm that the designed intelligent spectrum allocation scheme is a useful reference for multi-RATs spectrum management plans. 


\section{References}

[1] J. M. Peha, "Sharing spectrum through spectrum policy reform and cognitive radio", Proceedings of the IEEE, vol. 97, no. 4, (2009) April, pp. 708-719.

[2] S. Shellhammer, A. Sadek and W. Zhang, "Technical challenges for cognitive radio in the TV white space spectrum", Information Theory and Applications Workshop, (2009) February.

[3] FP7 project, "FARAMIR, Flexible and spectrum-aware Radio Access through Measurement and modelling in cognitive Radio system", Internet: http://www.ict-faramir.eu, ongoing.

[4] FP7 project, "COGEU, COgnitive radio systems for efficient Sharing of TV white Spaces in EU context", Internet: http://www.ictcogeu. eu/deliverables.html, ongoing.

[5] FP7 project, "QoSMOS - Quality of Service and MObility driven cognitive radio Systems", Internet: http://www.ict-qosmos.eu, ongoing.

[6] FP7 project, "SAPHYRE, ShAring PHYsical Resources mechanisms and implementations for wireless networks", http://www.saphyre.eu/publications/deliverables, ongoing.

[7] FP7 project, "SACRA - Spectrum and energy efficiency through multiband Cognitive RAdio", Internet: http://www.ict-sacra.eu, ongoing.

[8] C. Kloeck, D. Grandblaise, J. Luo and G. Dimitrakopoulos, "Multi-level spectrum auction through radio access", IEEE Mediterranean Electrotechnical Conference (MELECON), (2006) May, pp. 591-594.

[9] M. Li, X. Li, H. Ji and G. Dimitrakopoulos, "Virtual bidder group auction mechanism for dynamic spectrum access", 20th IEEE International Symposium on Personal, Indoor and Mobile Radio Communications, pp. 2797-2801, (2010) April.

[10] X. Zeng, Z. Feng, V. Le, Y. Xue and Y. Lin, "An auction based joint radio resource management scheme and architecture in a multi-operator scenario", IEEE Vehicular Technology Conference (VTC), (2008) May, pp. 2797-2801.

[11] FP7 "E3, End-to-End Efficiency", Internet: https://icte3.eu/project/deliverables/deliverables.html.

[12] 3GPP, "Feasibility study for orthogonal frequency division multiplexing for UTRAN enhancement", TR 25.892, V 6.0.0, 2004-06.

[13] G. Middleton, K. Hooli, A. Tolli and J. Lilleberg, "Inter-operator spectrum sharing in a broadband cellular network", IEEE Ninth International Symposium on Spread Spectrum Techniques and Applications, (2006).

[14] Z.-z. He, Z.-f. Zhang, C.-m. Chen and Z.-g. Wang, "E-commerce business model mining and prediction", Frontiers of Information Technology \& Electronic Engineering, vol. 16, no. 1, (2015), pp. 707-719.

[15] L.-m. Yang, W. Zhang and Y.-f. Chen, "Time-series prediction based on global fuzzy measure in social networks", Frontiers of Information Technology \& Electronic Engineering, vol. 16, no. 1, (2015), pp. 805-816.

[16] T. Beltramo, C. Ranzan, J. Hinrichs and B. Hitzmann, "Artificial neural network prediction of the biogas flow rate optimised with an ant colony algorithm", Biosystems Engineering, vol. 143, no. 1, (2016), pp. 68-78.

[17] X. H Peng and L. Q. Liu, "Wavelet Packet Neural Network Prediction Method Applied in the Gas Emission", Microelectronics \& Computer, (2016).

[18] Q. Hu, R. Zhang and Y. Zhou, "Transfer learning for short-term wind speed prediction with deep neural networks", Renewable Energy, vol. 85, (2016), pp. 83-95.

[19] J. Lai, J. Qiu, Z. Feng, J. Chen and H. Fan, "Prediction of Soil Deformation in Tunnelling Using Artificial Neural Networks", Computational Intelligence \& Neuroscience, vol. 8, (2016), pp. 1-16.

[20] S. Galeshchuk, "Neural networks performance in exchange rate prediction", Neurocomputing, vol. 172, no. C, (2016), pp. 446-452.

[21] S. L. A. Dixit, "Prediction of 2 Scrip Listed in NSE using Artificial Neural Network", International Journal of Computer Applications, vol. 134, no. 3, (2016), pp. 386-90.

[22] C. Lian, C. L. P. Chen, Z. Zeng and W. Yao, "Prediction Intervals for Landslide Displacement Based on Switched Neural Networks", IEEE Transactions on Reliability, (2016), pp. 1-13 


\section{Authors}

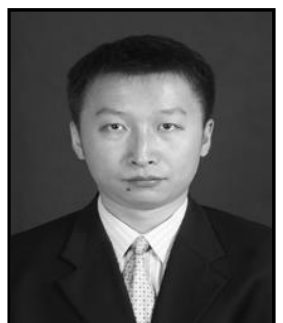

Yizhe Li, Senior Engineer of Wireless Technology Research Department of China Unicom Network Technology Research Institute. He received the M.S. and Ph.D. degrees from the Beijing University of Posts and Telecommunications. He joined the China Information technology designing and consulting institute in 2012 and is currently working on wireless network evolution and wireless new technology.

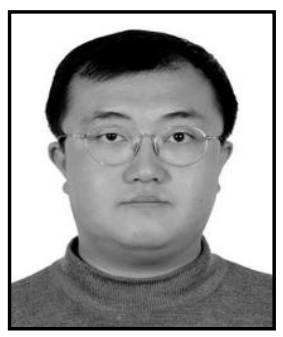

Zhonghao Zhang, Senior Engineer of Wireless Technology Research Department of China Unicom Network Technology Research Institute. He received the B. Tech. degree from Beijing Normal University and received the M.S. and Ph.D. degrees from Beijing University of Posts and Telecommunications. He currently works on wireless network evolution and wireless new technology.

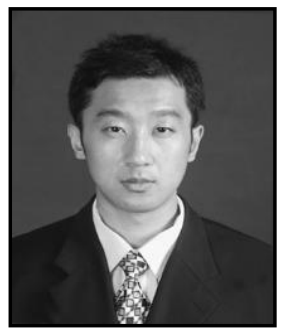

Lijun Chen, Planning Director of GU Planning \& System Design Department of ZTE company. He currently works on wireless network evolution and wireless new technology, including standardsetting, theoretical research and technical experiment.

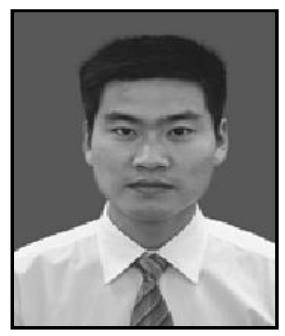

Fuchang Li, He received the M.S. and Ph.D. degrees from Harbin Engineering University. He joined the China Unicom Network Technology Research Institute in 2013 and currently is senior specialist of Wireless Technology Research Department. He currently works on mobile communication and fixed-mobile convergence, including standard-setting, theoretical research and technical experiment. He receive special government allowance from the State Council.

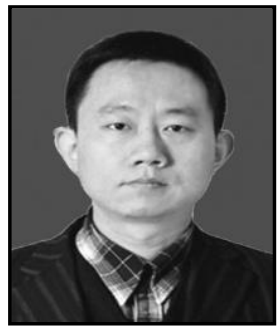

Yi Feng, Director of Wireless Technology Research Department of China Unicom Network Technology Research Institute. He Engaged in mobile communication network planning, research and designing and currently works for China Unicom IOT technology roadmap, development strategy and other research work. 
International Journal of Future Generation Communication and Networking Vol.10, No.4 (2017) 